\title{
Application Research of TMD on Low Frequency Vibration Control of Track Box Girder Structure
}

\author{
Xiaoyan $\mathrm{LEI}^{1}$, Xinya ZHANG and Kun LUO \\ Engineering Research Center of Railway Environment Vibration and Noise, Ministry of \\ Education, East China Jiaotong University, Nanchang 330013, China
}

\begin{abstract}
Vibration and noise problems of elevated track bridge structures are becoming increasingly prominent. In order to effectively control the low-frequency vibration response of the track box girder structure. Firstly, the controlled mode is confirmed through modal analysis of the box girder. The optimal stiffness, damping and attaching position of the tuned mass dampers are obtained based on the fixed-point theory and the identification method of equivalent quality for multi-degree-of-freedom system. Then, based on the vehicle-track-bridge coupling dynamic model, the control effectiveness of tuned mass dampers to low-frequency vibration of the track box girder structure under train moving load is discussed. The results show that the reasonable multi-mode modal tuned mass dampers combination can effectively suppress the low-frequency vibrations of the box-girder, and the vibration levels in the frequency bands $5-10 \mathrm{~Hz}$ and $20-31.5 \mathrm{~Hz}$ near the natural frequency are significantly reduced.
\end{abstract}

Keywords. Track box girder structure; vibration control; TMD; modal analysis

\section{Introduction}

Bridges are one of the important infrastructures for building railways and the key technology for railway transit. With the development of urban rail transit, the problem of vehicle-bridge coupling vibration has become more prominent, and the environmental vibration and noise problems caused by urban rail transit have also received more and more attention [1-3]. Carrying out the research on the vibration of urban rail transit bridges and finding reasonable measures to reduce the vibration of bridge structures are of great significance for reducing the low-frequency noise of bridge structures, protecting human physical health, and promoting the development of rail transit.

Tuned Mass Damper (TMD) is an effective low-frequency vibration control method for structures. It is often used for structural vibration control caused by earthquakes and winds. In recent years, it has been gradually used for bridge-induced vibration control. Yung-Hsiang Chen analyzed the effect of TMD on reducing the vibration of Timoshenko beams under moving loads [4]. Xiao Xinbiao studied the 
vibration mechanism and TMD vibration control of bridges under moving loads [5]. Gu Ping analyzed the effect of multi-tuned mass dampers (MTMD) on suppressing lateral vibration of railway steel truss bridge [6]. Zhang Xun selected $32 \mathrm{~m}$ double-line concrete simply supported box girder as the research object, and studied whether the installation of MTMD can achieve the dual effects of vibration reduction and noise reduction [7].

The above studies can show that the installation of TMDs on the bridge can effectively suppress the vertical resonance of the bridge, but the TMDs are designed just for a single natural frequency of the bridge, and the suppression frequency band is relatively narrow. Although the research of MTMD has improved the robust performance later, which adopts the central TMD to tune a certain order frequency of the structure, and the method of adding multiple TMD in a certain range of this frequency is still the vibration damping of the single mode of the structure. In view of the above research deficiencies, this paper designs the vibration absorber for multi-order modal natural frequencies. Based on the fixed-point theory, and the identification method of equivalent quality for multi-degree-of-freedom system, the TMD optimal design parameters for controlling the modal vibration of the box girder are determined to analyze the damping effect.

\section{Design of TMDs for Track Box Girder}

\subsection{Controlled Modal Analysis}

Through the constrained modal analysis of the track box girder structure, the mode contribution rate is used to determine the controlled mode of the box girder for vibration reduction. Firstly, the modal information of the finite element model of the elevated track box girder is calculated. The first 20 natural frequencies and mode contribution rates of the box girder are obtained as shown in Table 1.

Table 1.Modal frequency and mode contribution rate

\begin{tabular}{ccccc}
\hline \multirow{2}{*}{ Mode } & Natural frequency/Hz & \multicolumn{3}{c}{ Direction } \\
\cline { 3 - 5 } & 5.230 & $\mathbf{X}$ & $\mathbf{Y}$ & $\mathbf{Z}$ \\
\hline 1 & $\mathbf{6 . 1 0 3}$ & $\mathbf{0 . 0 0 0}$ & $\mathbf{0 . 7 8 4}$ & $\mathbf{0 . 0 0 0}$ \\
$\mathbf{2}$ & 9.520 & 0.000 & 0.000 & 0.941 \\
3 & 11.121 & 0.354 & 0.000 & 0.000 \\
4 & 11.987 & 0.000 & 0.000 & 0.000 \\
5 & 11.987 & 0.000 & 0.000 & 0.000 \\
6 & 11.987 & 0.000 & 0.000 & 0.000 \\
7 & 11.987 & 0.000 & 0.000 & 0.000 \\
8 & 13.215 & 0.000 & 0.000 & 0.000 \\
9 & $\mathbf{1 5 . 2 1 3}$ & $\mathbf{0 . 0 0 0}$ & $\mathbf{0 . 1 0 2}$ & $\mathbf{0 . 0 5 8}$ \\
$\mathbf{1 0}$ & 23.242 & 0.000 & 0.000 & 0.000 \\
11 & $\mathbf{2 3 . 7 6 2}$ & $\mathbf{0 . 0 0 0}$ & $\mathbf{0 . 1 1 4}$ & $\mathbf{0 . 0 0 0}$ \\
$\mathbf{1 2}$ & 23.988 & 0.000 & 0.000 & 0.000 \\
13 & 23.988 & 0.000 & 0.000 & 0.000 \\
14 & 23.988 & 0.000 & 0.000 & 0.000 \\
15 & 23.988 & 0.000 & 0.000 & 0.000 \\
16 & 31.432 & 0.010 & 0.000 & 0.000 \\
17 & 34.554 & 0.001 & 0.000 & 0.000 \\
18 & 36.019 & 0.000 & 0.000 & 0.000 \\
19 & 36.019 & 0.000 & 0.000 & 0.000 \\
\hline 20 & & 0.985 & 1.000 & 0.999 \\
\hline Sum of mode contribution rate & & & \\
\hline
\end{tabular}


It can be seen that the total contribution rate of the first 20 order vibration modes in the $\mathrm{x}, \mathrm{y}$, and $\mathrm{z}$ directions of the box girder is greater than $95 \%$, which means that the first 20 order vibration modes have met the need to determine the controlled modes of the box girder. Here we focus on the vertical dynamic response, so we only study the vertical (Y direction) mode of the box girder. It can be found that the Maximum contribution rate in the $\mathrm{Y}$ direction is the second order mode. The tenth order mode and the twelfth order modes are relatively larger. The 2 nd, 10th, and 12th order modes are confirmed as controlled modes with frequencies of $6.103 \mathrm{~Hz}, 15.213 \mathrm{~Hz}$, and $23.762 \mathrm{~Hz}$ respectively, whose shapes are shown in Figure 1.

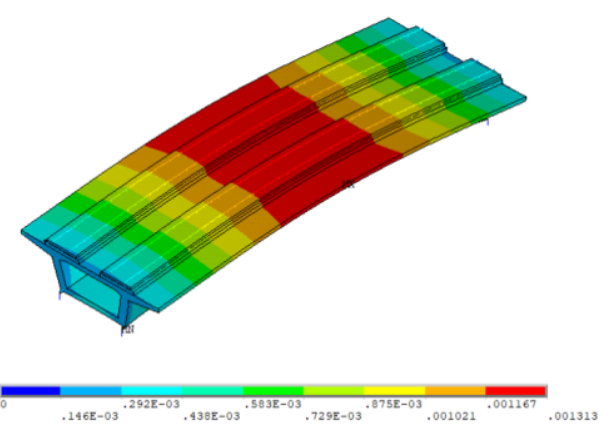

(a) 2 nd order mode $(6.103 \mathrm{~Hz})$
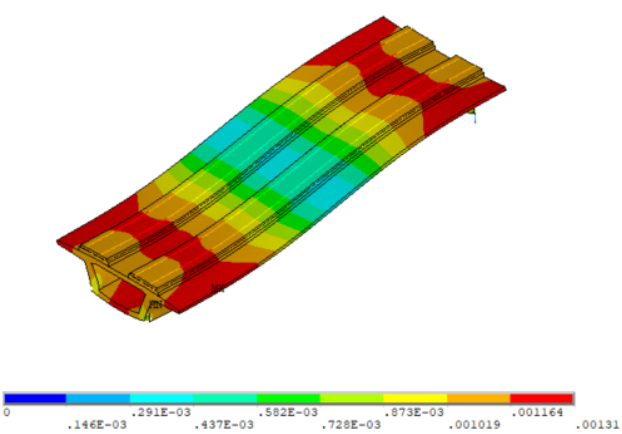

(b) 10th order mode $(15.213 \mathrm{~Hz})$
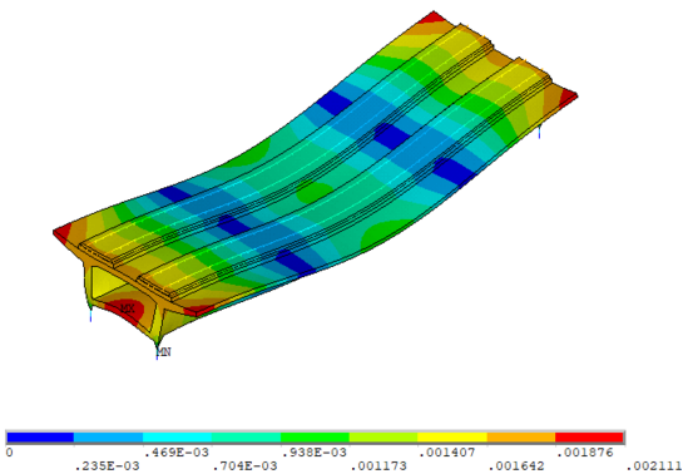

(c) 12 th order mode $(23.762 \mathrm{~Hz})$

Figure 1. Shapes of Controlled modes 


\subsection{Determination of TMDs Optimal Parameters}

In order to determine the optimal installation position and design parameters of TMDs, based on the above-mentioned multi-degree-of-freedom system equivalent quality identification method [8], the total energy and maximum modal displacement of the $i$ th mode of the box girder structure are calculated by the finite element analysis software ANSYS, and the equivalent mass of the $i$ th mode is calculated. In the analysis, when the mass ratio of TMDs for each order mode is selected as 0.01, 0.02, 0.03 and 0.04, the optimal design parameters of TMDs can be calculated as shown in Table 2, where $\alpha_{o p t}, \beta_{o p t}, M, m_{d}, K_{\text {opt }}$ and $C_{\text {opt }}$ represent the frequency ratio, damping factor, modal equivalent quality, quality of the $i$ th attached TMD, optimal stiffness and damping coefficient, respectively.

Table 2. Optimal design parameters of TMDs

\begin{tabular}{ccccccccc}
\hline Mode & $\begin{array}{c}\text { Mass } \\
\text { ratio }\end{array}$ & $\alpha_{\text {opt }}$ & $\beta_{\text {opt }}$ & $\boldsymbol{M} / \mathbf{k g}$ & $\boldsymbol{m}_{\mathrm{d}} / \mathbf{k g}$ & $\boldsymbol{f}_{\mathbf{s}} / \mathbf{H z}$ & $\begin{array}{c}\boldsymbol{K}_{\text {opt }} / \mathbf{\times} \mathbf{1 0} \mathbf{6}^{\mathbf{6}} \\
\left(\mathbf{N} \cdot \mathbf{m}^{-1}\right)\end{array}$ & $\boldsymbol{C}_{\text {opt }} /\left(\mathbf{N} \cdot \mathbf{S} \cdot \mathbf{m}^{-\mathbf{1}}\right)$ \\
\hline \multirow{3}{*}{$\mathbf{2}$} & 0.01 & 0.990 & 0.060 & 601852.0 & 6018.52 & 6.103 & 8.67 & 27417.6 \\
& 0.02 & 0.980 & 0.084 & 601852.0 & 12037.04 & 6.103 & 17.00 & 75993.8 \\
& 0.03 & 0.971 & 0.102 & 601852.0 & 18055.56 & 6.103 & 25.03 & 137146.2 \\
& 0.04 & 0.962 & 0.115 & 601852.0 & 24074.08 & 6.103 & 32.76 & 204256.5 \\
\hline \multirow{4}{*}{$\mathbf{1 0}$} & 0.01 & 0.990 & 0.060 & 719538.8 & 7195.39 & 15.213 & 64.43 & 132300.0 \\
& 0.02 & 0.980 & 0.084 & 719538.8 & 14390.78 & 15.213 & 126.28 & 226470.0 \\
& 0.03 & 0.971 & 0.102 & 719538.8 & 21586.16 & 15.213 & 185.94 & 408710.0 \\
& 0.04 & 0.962 & 0.115 & 719538.8 & 28781.55 & 15.213 & 120.71 & 301937.0 \\
\hline \multirow{3}{*}{$\mathbf{1 2}$} & 0.01 & 0.990 & 0.060 & 322827.3 & 3228.27 & 23.762 & 70.53 & 57259.7 \\
& 0.02 & 0.980 & 0.084 & 322827.3 & 6456.55 & 23.762 & 138.31 & 158708.1 \\
& 0.03 & 0.971 & 0.102 & 322827.3 & 9684.82 & 23.762 & 203.54 & 286420.3 \\
& 0.04 & 0.962 & 0.115 & 322827.3 & 12913.09 & 23.762 & 266.39 & 426575.7 \\
\hline
\end{tabular}

\section{Optimal Installation Location of TMDs}

It can be seen from the second-order mode shape diagram of the box girder shown in Figure 1(a) that, because the second-order mode of the box girder is vertical bending, the anti node of this order mode is located in the mid-span section of the box girder. And by observing the mode shape diagram of the second-order mode, it can be found that the anti node position of the second-order mode is close to the node position of the tenth-order mode. So According to the method of determining the optimal installation position of multi-mode modal TMDs [8], 2nd order modal TMD can be installed in the mid-section of the box girder. According to the calculation results, and by observing the 10th mode shape diagram of Figure 1(b), it can be seen that there are two maximum cross-sectional positions of the vibration deformation of the 10th order mode, which are $5.76 \mathrm{~m}$ away from the two girder end, and one of them can be arbitrarily selected as the optimal installation position of the 10th-order modal TMD. From Figure 1(c), we can see the anti node of the 12th mode of the box girder is located at the bearing section and mid-span section. Considering that the anti node of the 2 nd order mode is also located at the mid-span section of the box girder, in order to reduce the mutual influence of the modal coupling between the two order modal TMDs, Any anti node position of the bearing section can be arbitrarily selected as the optimal installation position of the 12th order modal TMD. The specific three order modal TMDs installation cross section is shown in Figure 2(a). In order not to increase the lateral rotation modal response of the box girder, the installation positions of the TMDs are all 
selected on the longitudinal center line, and the specific position is the top plate inside the box, which is shown in Figure 2(b).

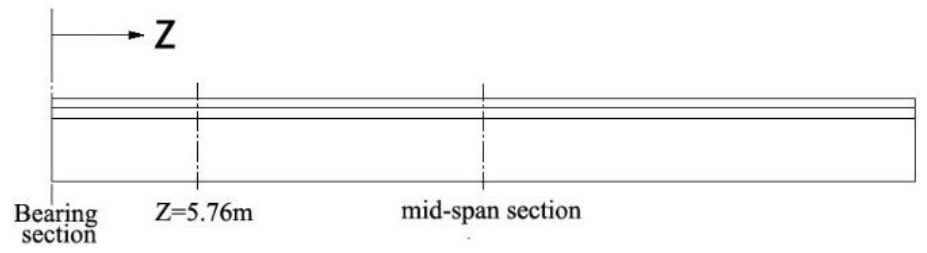

(a)Observation cross section

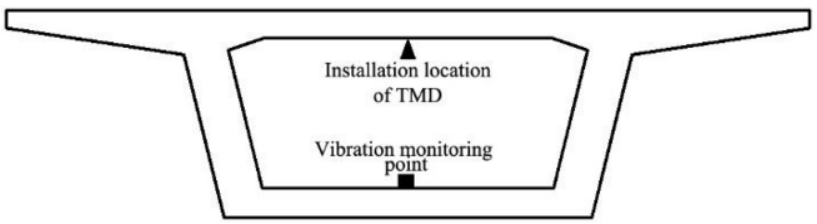

(b)Arrangement of characteristic points of each section

Figure 2.Optimal additional position for TMDs

A TMD vibration damping system is added to the box girder structure, and the mass element (Mass21) is selected to simulate mass block. The system's stiffness and damping characteristics are simulated by spring-damping element (Combine14).

\section{Vibration Reduction Characteristics of TMDs under Train Load}

This paper is based on the Metro B-type train established by the multi-body dynamics software UM, and uses the Flexible Railway Track in UM to establish the rail subsystem, and the rail is equivalent to the Timoshenko beam model. Simulate train running on track with UM and extract the wheel-rail force as shown in Figure 3. The type of track irregularity used in the simulation is the German high interference spectrum, and the wavelength range is $0.1-30 \mathrm{~m}$. Two trains are used for loading during calculation, and the running speed is $80 \mathrm{~km} / \mathrm{h}$.

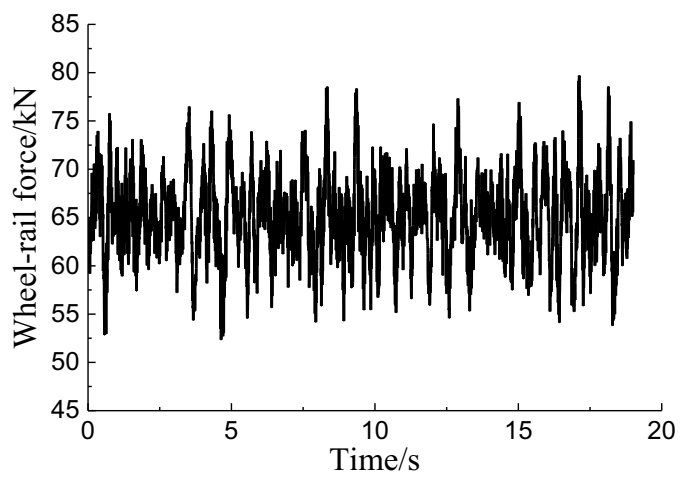

Figure 3.Time history curve of wheel-rail force

Think of the calculated wheel-rail force as a series of moving loads that change 
with time. By using the APDL language to write a DO cycle in ANSYS software and using the node loading method, the wheel-rail force is loaded on the box girder to realize the simulation of train passing bridge. Three order modal TMDs are added to the track box girder structure at the same time, and mass ratio is selected to be calculated by $0.03,0.02$, and 0.02, respectively. Figure 4, Figure 5 and Figure 6 show the vertical vibration acceleration time history and amplitude-frequency curves of the monitoring point sat bearing section, mid-span section and quarter section of the track box girder structure respectively. Then the damping effect is analyzed.

Figure 4(a), 5(a) and 6(a) show the vertical vibration acceleration of the track box girder structure caused by the train dynamic load before and after the TMDs controlling three order modes are added. From the comparison of time-domain curves, it can be seen from the figure that the maximum acceleration response amplitude of the bearing section, quarter section and mid-span section is significantly reduced after vibration reduction. And after the addition of TMDs, the overall damping of the track box girder structure increases, which makes the acceleration response of the train crossing the bridge decay faster. At the same time, the $\mathrm{Z}$ vibration levels before and after vibration reduction are shown in Table 3. It can be found that the vibration suppression effect on the mid-span section is the most obvious.

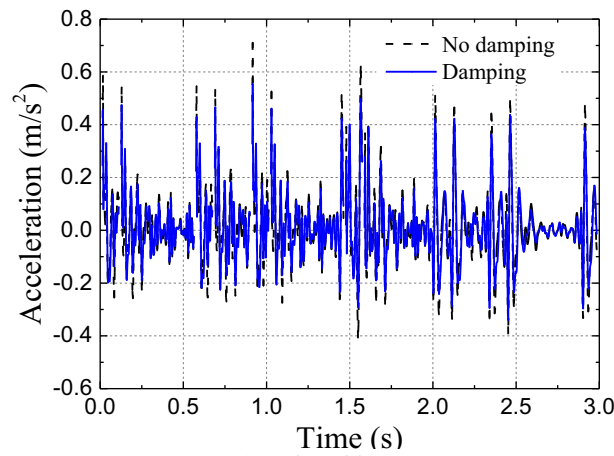

(a) Time history curve

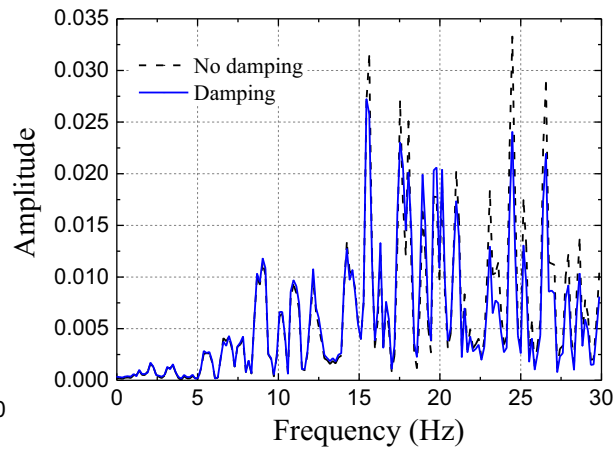

(b) Spectrum curve

Figure 4. Acceleration response of bearing section

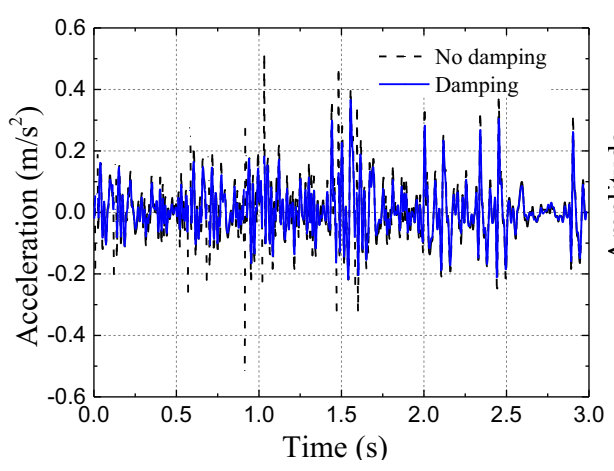

(a) Time history curve

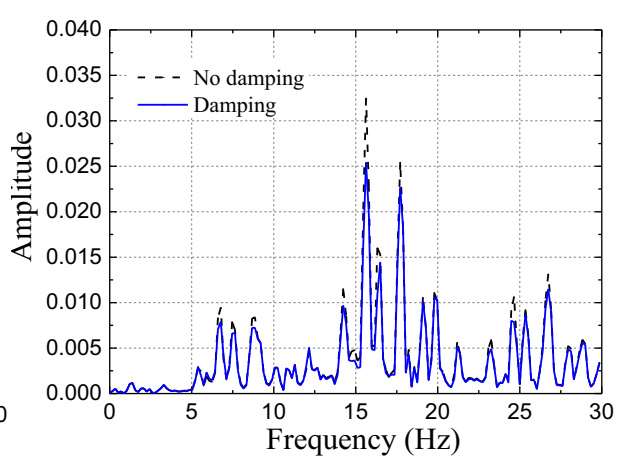

(b) Spectrum curve

Figure 5. Acceleration response of quarter section 


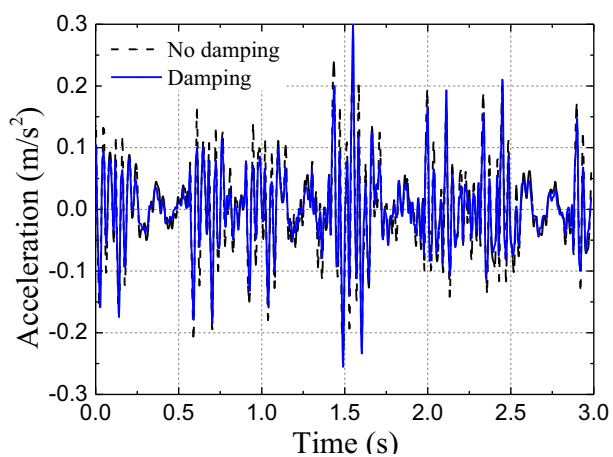

(a) Time history curve

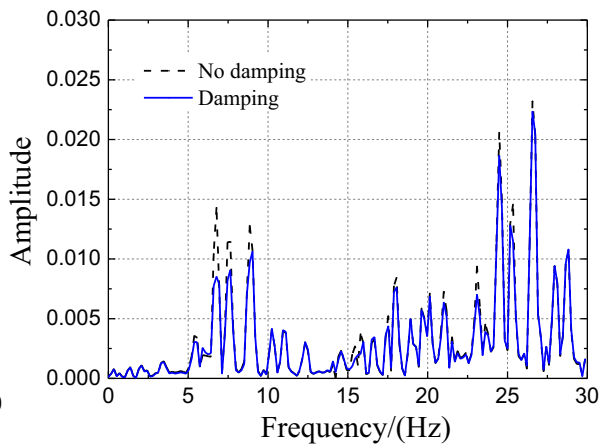

(b) Spectrum curve

Figure 6. Acceleration response of mid-span section

Table 3. $\mathrm{Z}$ vibration level before and after vibration reduction

\begin{tabular}{cccc}
\hline VAL/dB & Bearing section & Quarter section & Mid-span section \\
\hline No damping & 117.83 & 116.88 & 114.82 \\
Damping & 117.04 & 113.62 & 110.91 \\
Damping amount & 0.79 & 3.26 & 3.91 \\
\hline
\end{tabular}

It can be seen from the acceleration amplitude-frequency curve of the bottom plate at the bearing section that when TMDs is not added, the vibration of the track box girder structure caused by the moving load of the train has a larger response amplitude near the natural frequencies of $15.213 \mathrm{~Hz}$ and $23.762 \mathrm{~Hz}$. After the corresponding TMDs is added, the acceleration of the track box girder structure in the frequency band $15-30 \mathrm{~Hz}$ near these two natural frequencies is significantly reduced. From the amplitude-frequency curve at quarter-section bottom plate, it can be seen that in addition to the large peaks near the natural frequencies of $6.103 \mathrm{~Hz}, 15.213 \mathrm{~Hz}$, and $23.762 \mathrm{~Hz}$, the vibration acceleration of the box girder structure also stimulated new resonance peaks around $10 \mathrm{~Hz}$. With the addition of TMDs, the vibration acceleration response near the control frequency decreased significantly. It can be seen from the amplitude-frequency curve of the bottom plate at mid-span section that when TMDs is not added, the vibration acceleration of the track box girder structure caused by the moving load shows large peaks near the natural frequencies $6.103 \mathrm{~Hz}, 15.213 \mathrm{~Hz}$ and $23.762 \mathrm{~Hz}$. However, after adding TMDs, the vibration acceleration of the track box girder structure caused by the load is significantly reduced in the frequency range of $5-7.5 \mathrm{~Hz}$ and $15-28 \mathrm{~Hz}$ near the natural frequency, which indicates that TMDs effectively suppresses the vertical low-frequency vibration of the first few modes of the track box girder structure.

Before and after attaching multi-level TMDs, the $1 / 3$ octave curves of the vibration acceleration levels at the bearing section, quarter section and mid-span section of the track box girder structure are shown in Figure 7(a), 7(b) and 7(c). 


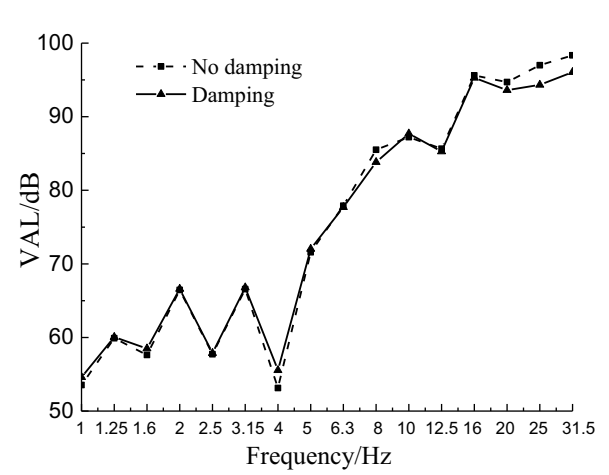

(a)Bearing section

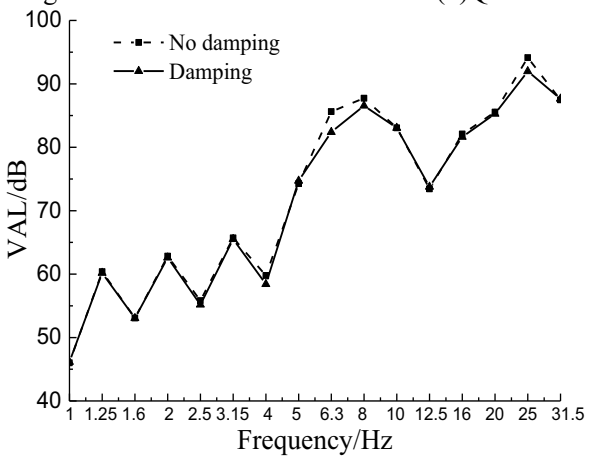

(c)Mid-span section

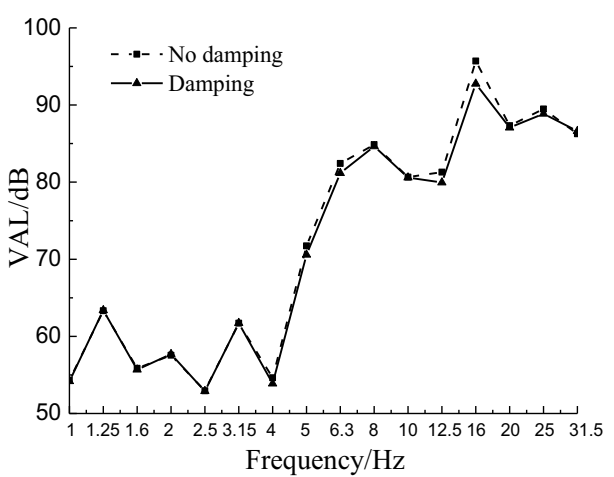

(b)Quarter section

Figure 7. Vibration acceleration level of each section

It can be seen from the Figure 7 that: the vibration acceleration levels of the bearing section in the $6.3-10 \mathrm{~Hz}$ and $16-31.5 \mathrm{~Hz}$ frequency bands are obviously different, and the vibration acceleration level can be reduced by $2-3 \mathrm{~dB}$; The vibration acceleration level of the quarter section in the $5-8 \mathrm{~Hz}$ and $10-25 \mathrm{~Hz}$ frequency bands is significantly reduced, and the maximum reduction is $1.3 \mathrm{~dB}$ and $3.0 \mathrm{~dB}$ respectively. The vibration acceleration level of the mid-span section is also significantly attenuated in the $5-10 \mathrm{~Hz}$ and $20-31.5 \mathrm{~Hz}$ frequency bands, which can be reduced by a maximum of $3.4 \mathrm{~dB}$. All these verify the effectiveness of using multi-order TMDs to control the low-frequency vibration of the track box girder structure.

\section{Conclusions}

(1) Based on the fixed-point theory, and the identification method of equivalent quality for multi-degree-of-freedom system, the optimal design of the additional TMD system for the elevated track box girder structure can be achieved.

(2) The largest contribution of the bridge vibration is the second order mode, and the contribution rate of the 10th and 12th modes is relatively larger, which can also be designed as controlled modes for additional TMD.

(3)Under the moving load, a multi-order modal vibration TMD damping system with a certain mass ratio combination is added to the track box girder structure. The low-frequency vibration of the track box girder structure is obviously suppressed, and the vibration acceleration levels of the frequency bands $5-10 \mathrm{~Hz}$ and $20-31.5 \mathrm{~Hz}$ in the 
vicinity of the natural frequency are significantly reduced.

\section{References}

[1] Thompson DJ, Jones CJC. A review of the modelling of wheel/rail noise generation. Journal of Sound \& Vibration, 2000, 231(3):519-536.

[2] Persson Waye K, Rylander R. The prevalence of annoyance and effects after long-term exposure to low-frequency noise. Journal of Sound and Vibration, 2001, 240(3):483-49.

[3] Waye KP. Effects of low frequency noise and vibrations: environmental and occupational perspectives.2011.

[4] Chen YH, Chen DS. Timoshenko beam with tuned mass damper to moving loads. Journal of Bridge Engineering, 2004, 9(2):167-177.

[5] Xiao XB, Shen HM. Vibration and the TMD control of bridges under moving loads. Journal of Vibration and Shock, 2005(02):58-61+149.

[6] Gu P, Wang M, Wu DJ. Damping suppression of lateral vibration of existing steel truss railway bridges using TMD. Journal of the China Railway Society, 2005(02):85-89.

[7] Zhang X, Li XZ, Liu QM, et al. Structure-borne noise control with MTMDs for a High-speed Railway Simply supported Box-Girder Bridge[J]. Journal of Vibration and Shock, 2013, 32(13):194-200.

[8] Zhang LQ, Zhu SY, Cai CB, et al. Application of dynamic vibration absorber in low frequency vibration control of floating slab tracks. Engineering Mechanics, 2016, 33(09):212-219. 